\title{
Application of Blockchain Technology in Cultural and Creative Design Education
}

\author{
https://doi.org/10.3991/ijet.v16i04.15233 \\ Jun Liu, Tiejun Zhu $(\bowtie)$ \\ Anhui Polytechnic University, Wuhu, China \\ ztj@ahpu.edu.cn
}

\begin{abstract}
Blockchain technology can realize an openness, transparency, non-tampering, non-forgery and the traceability of all information in the system without the endorsement of a third party, which can make up for the old and new problems in the field of cultural and creative design education. This paper first analyzes the technical principles of blockchain and the deficiencies of online teaching and cross regional teaching in cultural and creative design education, and then applies blockchain technology in learning resource management, learning process tracking, learning evaluation, learning path shaping, and teaching management and assessment. It is concluded that blockchain technology can effectively optimize the teaching structure and management in cultural and creative design education, and promote the design education field to adapt to the contemporary development. Finally, it puts forward the future prospects.
\end{abstract}

Keywords—Blockchain, Cultural and creative design, Education, Application

\section{$1 \quad$ Introduction}

\subsection{Blockchain overview}

On January 3, 2009, the Genesis Block with serial number 0 was born. Six days later, the block with serial number 1 appeared and connected with the Genesis Block to form a chain, thus the blockchain was born. Computer experts describe it as a decentralized distributed ledger database. Each block is like a hard disk, which saves all the information and encrypts it in the block through cryptography technology. Without mutual trust, blockchain technology can provide a decentralized and transparent data storage mode by using the mutual cooperation between blockchain network nodes based on consensus mechanism and distributed P2P network protocol for communication. The data stored in blockchain is packaged into blocks to form a chain structure and copied to each node, so it's called distributed public account book. With the cooperation of cryptography, hash technology, consensus algorithm, smart contract and other related technologies, the data in the blockchain is easy to verify and difficult to be maliciously modified and completely destroyed. 
Table 1. Blockchain technology platform architecture

\begin{tabular}{|l|l|}
\hline \multicolumn{1}{|c|}{ Application layer } & \multicolumn{1}{c|}{ Programming interface, user interface, various applications } \\
\hline Intelligent layer & Script, virtual machine, programming language \\
\hline Consensus layer & Consensus algorithm, excitation mechanism \\
\hline Network layer & $\begin{array}{l}\text { P2P network, Communication mechanism, } \\
\text { Verification mechanism }\end{array}$ \\
\hline Data layer & Data Block, Chain structure, Data model, File storage \\
\hline
\end{tabular}

\subsection{Blockchain features}

Blockchain has four basic characteristics: The first is that information cannot be tampered with. Users jointly maintain the security of data on the Internet to ensure that it cannot be tampered with. Once the information is validated and added to the blockchain, it is stored permanently. Unless more than $51 \%$ of the nodes in the system can be controlled at the same time, the modification of the database on a single node is invalid, so the blockchain's data stability and reliability are extremely high.[1]

The second is to become decentralized and distributed, and it is important to note that distributed is a physical center, not a management center. For example, largevolume transactions are carried out simultaneously, and the decentralized approach will save resources, make trading autonomous and simplistic, and avoid the risk of being manipulated by the central agent.

The third is privacy and supervision. From the current construction of the blockchain, these two seemingly contradictory points of privacy and supervision can be integrated. Because the exchange between nodes follows a fixed algorithm, the rules of the program in the blockchain will automatically determine whether the activity is valid. Hence, their data interaction is not trustworthy, and therefore the two sides of the transaction can generate mutual trust without disclosing their identities.

The fourth point is the smart contract, which can realize the legal application based on the blockchain contract rules, and the top-level governance node can formulate the smart contract.[2] A smart contract is a contract written by code and automatically executes the contract signed by both parties in the contract when the conditions are met. Smart contracts are widely used in many fields. 


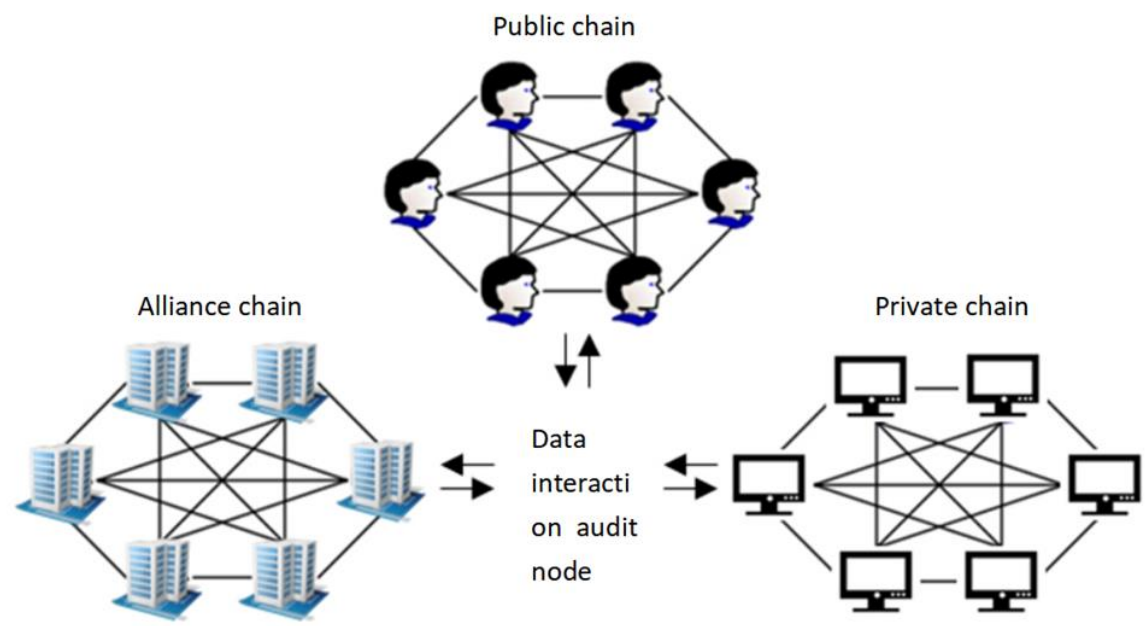

Fig. 1. Teaching data sharing blockchain architecture

The current dilemma hindering the development of blockchain projects is that, in addition to the application of this technology in storage, traceability and other aspects, compared with the traditional centralized scheme, there is no obvious advantage, and no applicable business model can be found. Although in the practical application, the development of blockchain projects has declined, it is fortunate that many scholars and experts now focus on basic fields such as medical treatment and education, and actively accumulate relevant experience, technology and develop new application models, believing that blockchain technology will inject fresh vitality into the traditional design education field.

\section{The New Situation and Main Problems of Lifelong Education and Cross Regional Education in The Field of Cultural and Creative Design Education}

Creative industries and cultural and creative brands, as an important driving force for the development of the economy and the promotion of culture, have become the representative of the country's soft power. Cultural and creative product design finds cultural elements of human history from the perspective of cultural and artistic exchanges, which is also closely related to the social and cultural vision of the times. [3] At the end of 1990s, the design education in Chinese universities realized the importance of the connection between cultural and creative design and the revival of traditional culture, and through the design practice, those traditional handicrafts, operas, museum cultural relics, etc. which had been forgotten by people, were displayed in front of people again, which not only gained the public's attention, but also led to the establishment of related industries, and produced huge economic performance. Facing the new era of design development, cultural and creative design education in Chinese 
universities should actively interact with foreign design colleges, and carry out online teaching with the help of the technical advantages of the Internet, so as to bring Chinese cultural and creative design unto a new platform. However, there are some new problems with the rapid development of cultural and creative design education, because the traditional design classroom system has not been able to adapt to the changes in teaching content and design direction.

\subsection{The contradiction between the development of design lifelong education and education centralization under the Internet}

Lifelong education refers to the sum of all kinds of education people receive in all stages of their lives, including all stages and modes of education system, including school education, social education, formal education and informal education.[4] The market demand of cultural and creative products and the designer's self-requirements make lifelong education more and more important. The direction of the wind in the field of cultural and creative design has been changing, which requires of designers to supplement knowledge all the time and make targeted feedback in the face of the ever-changing market, including a series of changes in their own design system, such as design thinking, design inspiration, design style, etc. The emergence of this situation makes the field of cultural and creative design education more and more active. The former school-centered design education behavior has gradually shifted to education and training institutions, designer studios, network design teaching, enterprises and other fields. The timeliness and foresight of these teaching methods are unmatched by classroom education, because the materials come from first line design practice, people upload or share the education resources to the network platform, and then the registered identity can participate in the class, and interact with the teachers in the network classroom, making the teaching process simple and efficient.

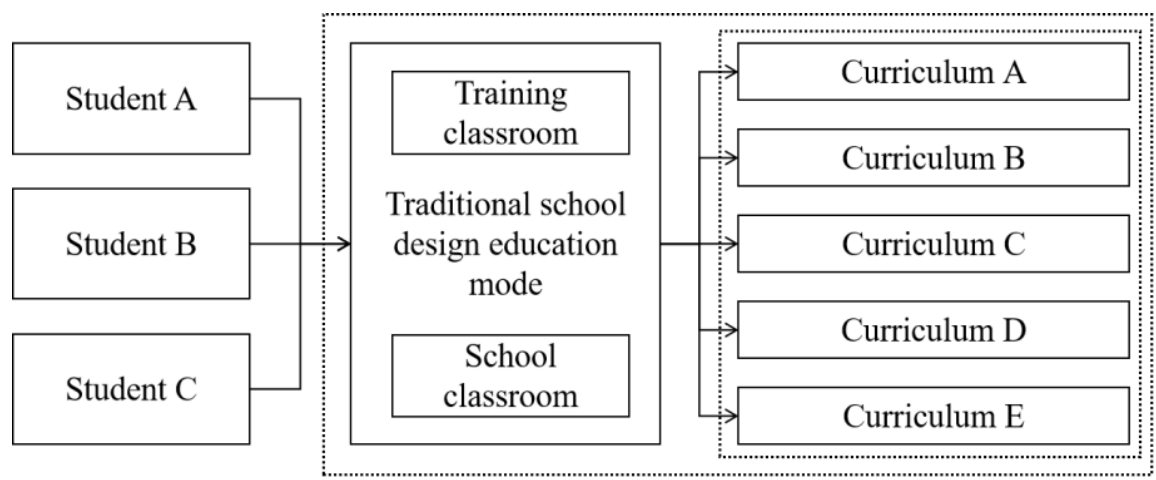

Fig. 2. Traditional centered design education mode

However, it is worth pondering that a large number of design education resources in China are placed in a closed place like the school. It is difficult for people who are separated from the student status to have the opportunity to understand and learn 
again. The traditional education mode makes it difficult for most people to have equal right to education, narrowing lifelong learning in the field of design. On the other hand, the network education resources are uneven, some are good and others are bad, which increases people's learning time, cost and unforeseen learning burden.

\subsection{The contradiction between the popularization of cross regional design teaching, qualification certification and education evaluation}

Nowadays, design communication is becoming increasingly popular all over the world, and its modes of communication are also increasing in diversity. There are four main modes of design communication in Chinese universities: visiting, expert lecture, seminar, and cross regional teaching communication. The cross regional teaching method can provide a good innovation platform for teachers and students. Through this platform, reasonable use of the differences between design colleges and universities can expand the scope of student-innovative thinking, promote the depth of thinking, and facilitate the validation and revision of their own design curriculum system.

${ }^{[5]}$ Particularly in the internationalization of college and university teaching, foreign exchange has been expanding year by year, which has given birth to many cases of cooperative education of product design specialty. Through the blend of eastern and western culture via design behavior, design literacy and aesthetics have been improved while opening up the horizon. But back here in China, this learning experience can hardly be a powerful proof for students' learning skills, literacy and teaching results. Because traditional educational institutions lack effective methods, resources and abilities to validate learners' knowledge, skills and results, it is again difficult to manage and validate learners' learning activities, processes and results concurrently. This situation makes the learning experience and cultural and creative design achievements of cross regional teaching staff greatly discounted in the interviews of enterprises and government departments, weakening their competitiveness in job interviews and employment.

At present, the modernization of art design and art design education in China has entered a critical period.[6] Development of information technology and the upgrading of teaching equipment have changed the structure of traditional "centralized" design education. For this reason, other disciplines such as economics, biology, computer, media, among others, are actively modifying their teaching modes, increasing their reliance on Internet technology, social software self-Media, network new media and other active "decentralized" activities. However, the traditional cultural and creative design classroom based on fixed location and fixed time, which implies considerable time and transportation costs, has become a very expensive and relatively inefficient way of knowledge dissemination.[7] The conventional design education has lagged behind the development of the times. It has severe contrast with the new development concept of modern education, which makes the reform and progress of design education difficult. The focus of teaching is on textbooks and teaching sites are schools or classrooms, which makes the design inspiration and concept lose its ground with the spirit of the times and futurism. Cultural and creative design education is thus running counter to the future. 


\section{The Application of Blockchain Technology in Cultural and Creative Design Education Industry}

\subsection{Learning resource management}

Art design and education are the first to involve information technology in reform and opening up. Today's derived MOOC, flipped classroom and other teaching methods are inseparable from the foundation of art design and education. ${ }^{[8]}$ Facing the new teaching reform in the field of design education, the main body of the curriculum design, such as MOOC and flipped classroom, is still based on the traditional textbook content and teaching form of design education. This also leads to the inability of cultural and creative design teaching to attract students' attention and stimulate their design creativity. The main reason is that: the traditional design teaching work is highly one-sided; the corresponding teaching content is overly concerned with the textbook knowledge, the lack of contact with the cultural and creative product design practice, and the lack of awareness of the cultural and creative product design background and the history involved. Therefore, in this link, we can develop the corresponding cultural and creative teaching resources, establish the teaching structure system, connect the intangible cultural heritage masters and intangible cultural heritage protection platform, and update the corresponding teaching information and content online real-time. Teachers and students, enterprises and institutions can download the resources as learning reference and design materials, as well as upload their own works, design inspiration and learning information, so as to improve the learning resource management structure, enrich the classroom content and teaching mode, as well as stimulate students' learning creativity and attention.

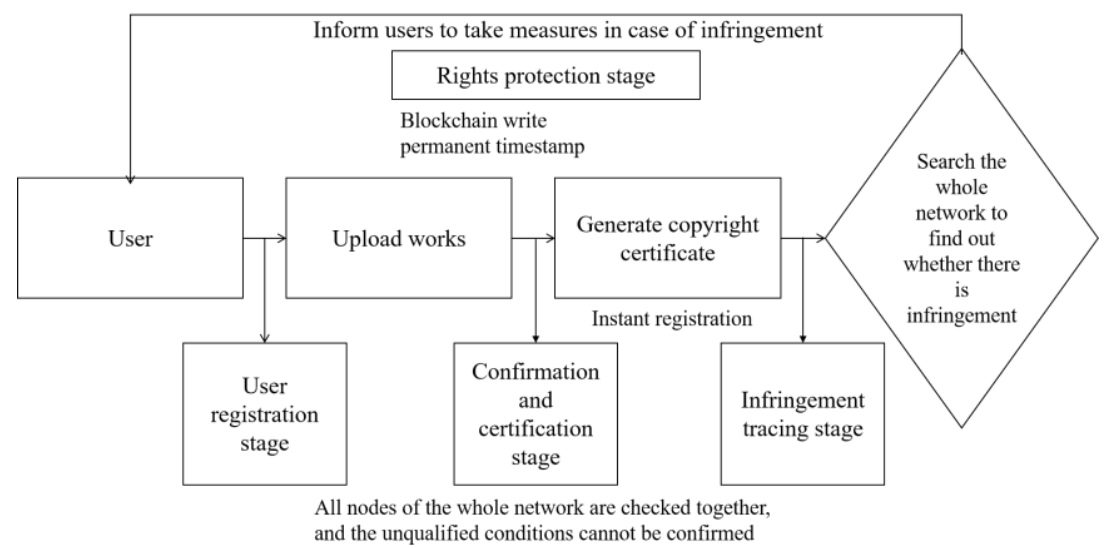

Fig. 3. Intellectual property rights confirmation process of blockchain cultural and creative products 


\subsection{Learning process tracking}

Colleges and universities can fully integrate the data management of students' personal electronic status with blockchain technology. In colleges and universities, information technology is used to record students' elective subjects, course scores, practice competitions, etc. as data blocks, and students' learning status and learning achievements are recorded through blockchain technology. With students' learning experience and design activities, each corresponding education blockchain will continue to increase and will not be deleted on the background system, becoming the learning mark following student life. With the traceable characteristics of blockchain technology, the copyright of teaching practice results and design works is also protected invisibly. Once there is an infringement, the rights can be protected immediately. Where third parties including education departments, enterprise companies, etc. need to verify the learning data of students, they could easily obtain student curriculum credits, academic certificates, design works, design awards, etc. inside or outside the school.

\subsection{Learning assessment}

It is the most effective way to evaluate students' learning progress with practice and competitions in cultural and creative design and education, but the existing problem is that the evaluation may be opaque and not objective, and the evaluation results of students' design and learning may be changed consciously due to influence from external factors. Because of the differences among evaluators, schools, regions and even countries, it is difficult for traditional educational institutions to effectively evaluate and certify students' learning process and results objectively, fairly, automatically and accurately. The teaching evaluation based on blockchain technology can be transformed into the certification of students' learning achievements, and the corresponding feedback of students' learning effect can help the curriculum system to find shortcomings and further improve. From the perspective of security, the use of blockchain technology can ensure anonymity and provide multiple signature technology for each group to ensure information traceability. In the data layer architecture of the doublelayer chain, the main chain information is generated into the information in the subchain block. As long as the integrity of the main chain full function node is guaranteed, the malicious attack of the chain block can be protected. The data stored in the block mainly includes course name, design practice project, course weight and other subjects. Besides, it also includes the qualification conditions for graduation requirements as one of the evaluation basis, and design course learning as the output value of the result learning. It is determined by comprehensive evaluation of students' learning achievement, learning process and learning evidence through quantitative and qualitative methods. In this way, the traditional evaluation based on achievement and credit has become the evaluation based on the ability and index of graduation requirements, and at the same time, under the condition of using consensus mechanism, it ensures the persuasiveness of the evaluation results, because the evaluation is no longer limited to the subjective will of teachers. At present, some colleges and universities have 
published the teaching materials of entrepreneurship and innovation education which are popular with students, and produced more practical and mature teaching methods and evaluation standards.[9] It can be said that applying blockchain technology to learning evaluation can effectively make up for the shortcomings of learning evaluation in the current design education field.

\subsection{Learning path shaping}

At present, in the process of personalized recommendation of design education, when learning resource feature matching is insufficient, it is necessary to collect, analyze accurately and allocate education resources reasonably.[10] This leads the design education in colleges and universities to change the learning environment of students according to the relevant problems of the course, enabling students to acquire creative passion and abilities.[11] The basic courses of cultural and creative design education in colleges and universities in China are generally arranged in the period of freshman and sophomore years. Through the two-year design basic courses, students' design literacy, innovative thinking and corresponding design basic knowledge are cultivated. However, in the period of junior year, it is necessary to further distinguish the specific design direction. At this time, blockchain technology can be used to help learners shape their own design learning path through different types of smart contracts, for example, some students take interest in traditional handicraft cultural and creative design, while other students are fascinated by calligraphy and painting cultural and creative design. The intervention of blockchain technology thus enables students to clearly find their own expertise and learning interests. At the same time, it also helps teachers to guide students' learning, so as to find out the design path plan suitable for students and to realize the tracking of students' learning process and further learning motivations.

\subsection{Teaching management and assessment}

In the new era, great changes have taken place in the requirements of various colleges and universities for the cultivation of students in the direction of cultural and creative design. The most important change is that the focus has shifted from the design results and curriculum achievements to the personalized development of students, the cultivation of innovation awareness, practical ability training and other aspects, which weakens the evaluation leading role of examination results, and brings new work requirements to the traditional teaching management. In the traditional teaching management, teachers need to record the students' classroom performance and fill it into the students' paper files. Then, in the middle or end of the course, they need to analyze the students' learning situation and make personalized teaching strategies. This method cannot give feedback to students' learning changes in time while increasing teachers' workload, which renders the interaction of teachers and students ineffective. The blockchain technology can make up for these shortcomings of teaching management, fully integrate the information on students' personal electronic status 
with the blockchain management technology through technical means, and create an efficient and easy-to-manage education management platform.

For example, the construction of a cultural and creative design education management platform based on blockchain technology, in which the body of the chain mainly includes the government education department, the teaching management departments of colleges and universities, the universities' students' offices, the design studio and the design school. The positioning of each subject in the alliance chain is as follows: the teaching management departments of colleges and universities create the alliance chain, authorize the added nodes with trust and give the required permissions, and operate and maintain the data of teachers' teaching and students' learning on the blockchain together with different subjects. The education department of the government is responsible for supervising the behaviors of all subjects on the chain, avoiding problems in time, and participating in the maintenance of the blockchain. In case of disputes, the education department is responsible for solving them. The management teacher is responsible for uploading and recording all files of the students during the school period. The students' office of the university must confirm and endorse all the contents filled in by the management teachers to ensure the authenticity and validity of the student data files. The design studio is responsible for recording the design and teaching activities participated in by students on the chain, including the content, time, achievements and other information. The design studio must ensure the authenticity and effectiveness of the data recorded on the chain. The design school is the user of blockchain information, jointly maintaining and recording student file information. It can view the trusted student file information recorded on the blockchain through permission application, in order to realize accurate assessment of students.

\section{The Main Problems in The Application of Blockchain Technology to Cultural and Creative Design Education}

\subsection{Complex operation technology and user information security}

Although blockchain technology has many application advantages in cultural and creative design education, its specific operation method is not easy to learn. Many design majors may not be able to easily learn and understand the corresponding file download and upload methods, creating obstacles for students' independent learning. For colleges and universities, many teachers in charge of student information management need training and ability certification before they can work, which also affects the progress of student management. Secondly, due to the need for openness and transparency of user information, many teachers and students will have dire concerns about the security of their personal identity information. For example, many harassing mobile SMSs and phishing emails steal personal information through network technologies, which necessitates the optimization of the security management of the blockchain technology in cultural and creative design education. 


\subsection{Manual data inputting and user system compatibility}

There may be some phenomena such as content falsification, cheating in scores and input errors when data upload is done manually. In blockchain technology, once data is uploaded, it cannot be modified, as the change of data in one block will result in changes in the corresponding blocks within the chain, and this increases the usage risk of blockchain. At the same time, some operating systems are not compatible with the software platform, which makes it difficult to upload and/or download data. Resultantly, it is more difficult to achieve a seamless flow of information between and/or among users in the blockchain, and this affects the optimal application of the technology.

\subsection{Customization of relevant technologies}

The focus of education should not be on improving the economic interests of the school, but paying more attention to training high-quality cultural and creative design talents to meet the requirements for social development. This is determined by the cultivation of talents with innovative thinking, the cultivation of practical student abilities, and the cultivation of team-spirited students in design education.[12] Therefore, it is required that the relevant staff innovates and optimizes the blockchain technology operation methods to better meet the needs of the cultural and creative design education. Hence, not only should blockchain be a basic technology of bitcoin, but also to be customized to reasonable problem settings and technical structural reforms and innovations.

\section{Conclusion}

Through the exploration of blockchain in the field of cultural and creative design, this paper demonstrates solutions to the problems in the current teaching, education management and student evaluation of cultural and creative design, such as the difficulty in qualification certification and the opacity of teaching evaluation. It provides technical support for lifelong teaching and cross regional teaching, and as well promotes the development of lifelong education with technology in the cultural and creative design field. It tries to break the traditional rigid mode of centralized teaching, so that people can remotely and independently enjoy the right to education. In cultural and creative design education, learning resource management, learning process tracking, learning path shaping and other new upgrading of the structural and management modes of the blockchain technology helps avoid teaching traps and improve work efficiency.

Nowadays, the art design major pays more and more attention to the cultivation of students' creative thinking and practical abilities, so as to improve the employability of students as well as bringing more students to the art design school.[13] On the one hand, the further optimization and algorithmic innovation of blockchain technology can better assist teaching to improve students' thinking and practical ability in cultural 
and creative design, ensure students' relevant interests in online teaching and cross regional teaching, and provide sufficient and adequate learning materials. In promoting employment, it can further help students to connect more widely with employers. Because of its technical characteristics, it can be well maintained and secured to fight against piracy, design infringement, etc., and can also facilitate online teaching payments, cultural and creative works copyright maintenance, teaching courseware copyright determination, and so on. On the other hand, learning from the idea of blockchain distributed governance in the field of education, the world wide web is a natural distributed governance platform for educational resources, which can be combined with user needs to achieve an effective distributed governance.[14] Blockchain technology can more effectively use the materials of cultural and creative design education to provide more and better design teaching services for students, reduce the energy and time of users in identifying the relevant learning resources, and further improve the quality and efficiency of education. In the future, there will be more cultural and creative design works and online courseware based on blockchain technology, which will further promote the application of blockchain technology in cultural and creative design education.

\section{Acknowledgement}

This work is supported in part by the key online teaching reform research project of Anhui Polytechnic University "Research on online implementation and management of international students' intelligent classroom and practical teaching in case of outburst of large-scale epidemic(2020xsjyxm08); 2018 Anhui provincial quality engineering project "Sino-foreign cooperation training project" (2018zwpy001); Zhongjiang scholar talent project and Creation Group Project "Local Culture and Creative Design Research Group" of Anhui Polytechnic University(S03011).

\section{$7 \quad$ References}

[1] Sina.com, http://www.sohu.com/a/274020698_492538, last accessed 2019/12/25.

[2] Zhiming, Z. (2019). Blockchain technology and development.

[3] Chuan, C. (2019). On the teaching mode of cultural and creative design course in Colleges and Universities Based on the cooperative development of Museums. Journal of Green Science and Technology, 19, 272-273.

[4] Shanshan, Z. (2019). On the theory of transforming learning under the idea of lifelong education. Journal of Changchun Normal University, 38(11), 158-160.

[5] Youcai, W., Yu-an, S., Zhengyi, C. (2012). Exploring the Influences on Design Innovation from Trans-Regional Design Exchange Programs. Academic Exploration, 07, 176-178.

[6] Dan, X. (2019). The current situation and development prospect of art design major in Colleges and universities in China. Higher Education Exploration, 08, 141.

[7] Fengmin, J., Lingyun, H. (2019). Exploration on the Teaching Reform of Basic Courses of Finance Theory under the Impact of "Decentralization" of Internet Development. Research of Finance and Education, 32(01), 76-80. 
[8] Jin, H., Yunfeng, Z. (2019). Thoughts on the integration of production and education of art design education in Higher Vocational Colleges of Hunan Province. Yihai, 11, 101-103.

[9] Cheng, C. (2019). Analysis on the current situation and problems of innovation and entrepreneurship education in higher vocational colleges-Taking landscape architecture design as an example. Xiandai Horticulture, 20, 229-230.

[10] Jiao, H., Wenlan, Z. (2019). Exploration on Construction Path of Online Course Resources for Personalized Learning. Teaching \& Administration, 33, 18-20.

[11] Zhi, L. (2019). On the development of art design major in Higher Education. Jushezazhi, $21,127$.

[12] Shumin, L., le, Z., Xiaopeng, P. (2019). Research and development suggestions on the current situation of art design education in Colleges and universities in China. Art Education Research, 21, 146-147.

[13] Juyun, Y. (2019). Current Situation and Development Countermeasure of Art Design Education in Secondary Vocational School. On contemporary teaching and research, 09, 133.

[14] Xiang, F. (2019). Education resources governance in the age of Education Informationization 2.0:ideas and paths. Distance Education in China, 40(12), 12-23,33,92.

\section{Authors}

Liu Jun is a graduate student of Anhui Polytechnic University, Wuhu, China.

Zhu Tiejun is a full professor, Zhongjiang scholar and graduate student supervisor of Anhui Polytechnic University, Wuhu, China.

Article submitted 2020-04-27. Resubmitted 2020-11-05. Final acceptance 2020-11-05. Final version published as submitted by the authors. 